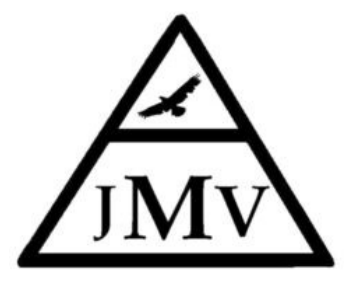

\title{
A review of hospital based ventilator malfunctions reported to the FDA in 2021
}

Stephen Tunnell BAAS, RRT

DOI: https://doi.org/10.53097/JMV.10037

Cite: Tunnell S. A review of hospital based ventilator malfunctions reported to the FDA in 2021. J Mech Vent 2021; 2(4):142-146.

\begin{abstract}
Ventilator care is synonymous with Intensive care. These devices are electromechanical and as such can fail. Most failures are without patient incident, injury, and harm.

The FDA requires manufacturers who learn of malfunction, injury or death while operating their product to report to the agency via the Manufacturer and User Facility Device Experience database.

I reviewed 500 recent events reported to the FDA and found an increasing trend from 2020 to 2021 in hospital ventilator malfunction reports. Examination of these reports is critical to assuring quality ventilator care.

The author concluded that intensive training on the device characteristics and feature and a more rigorous examination of ventilator performance between patients may assist in reducing device malfunctions.
\end{abstract}

Keywords: Mechanical ventilation, Ventilator malfunction, FDA

Author

Stephen Tunnell BAAS, RRT Chief Science Officer, Lungtreater CRO

Corresponding author: CRO@Lungtreater.com

Conflict of interest/Disclosures: None

Funding: None

Journal of Mechanical Ventilation 2021 Volume 2, Issue 4

This open-access article is distributed under the terms of the Creative Commons Attribution Non-Commercial License (CC BY-NC)

(http://creativecommons. org/licenses/by-nc/4.0/), which permits reuse, distribution and reproduction of the article, provided that the original work is

properly cited and the reuse is restricted to noncommercial purposes. For commercial reuse, contact: editor@journalmechanicalventilation.com 


\section{Introduction}

Ventilators provide life-sustaining therapy within hospitals and more recently the public has become aware of the importance of ventilator care, particularly in the intensive care ward. Ventilators have a wide-ranging set of specification and requirements allowing them to treat neonates to adults that make their use restricted to professionals. In the US Respiratory Therapists are the primary operating professional.

The US Food and Drug Administration (FDA) clears mechanical ventilators for interstate commerce thus allowing manufacturers to market their products in the US. Additionally, the FDA compels manufacturers and requests of users to report adverse device events to them.

The FDA houses these reported events within the Manufacturer and User Facility Device Experience database, aptly called MAUDE. ${ }^{1}$ These events can be accessed easily via the Total Life Cycle Database, ${ }^{2}$ which contains pre and post market information on ventilators.

Regular use of these databases is important for manufacturers but also hold a treasure trove of data for physicians and caregivers. With Capital dollars scarce it is important for hospital administrators to be aware of ventilator device reports and any potential issues in candidates for purchase. Risk management staff may also be informed of potential hazards that some devices may pose within their installed base; and which may be easily mitigated via staff education or may require more complex interaction and service intervention with the manufacturer or their representative.

This information is important in creating a safe and effective approach to the application of mechanical ventilation care.

\section{Method}

We reviewed 500 reported ventilator device problem events in 2021 between October 15 and October 30 reported to the FDA and published in the Total Product Life Cycle (TPLC) data base for Continuous Use Facility Ventilators and regulated as product code CBK. Fully regulated by 21CFR868.5895 and identified as a continuous ventilator (respirator), which is defined by the FDA as "a device intended to mechanically control or assist patient breathing by delivering a predetermined percentage of oxygen in the breathing gas".

Adult, pediatric, and neonatal ventilators are included in this generic type of device classification.
It is important to note that other categories of ventilator devices exist, such as transport or Emergency use ventilator categories (product code: BTL) and were not examined here. These devices also have data available in the TPLC or MAUDE database.

Data gathered was sorted by manufacturer, brand name, and event type, which included death, injury, and malfunction.

Data was then compared to the current run rate of events per month to determine relationship to normative rates for malfunction reported events.

All data was then appraised for dominant events and significant trends. In the case of death or injury further review of the file was warranted to determine any relevant implications.

\section{Findings}

500 Ventilator events (CBK - ICU ventilators) were recorded into the database representing 15 working days in October. Annualizing this rate results in 12,167 malfunction events per year. The previous months had run rates averaging 1,005 per month.

2020 had a total of 11,096 events so this is an increase of $9.6 \%$ increase in ventilator malfunction events.

Table 1 indicates the top ten reported event device problems during this 15-day period.

This top 10 represents $80 \%$ of the reported events.

Table 2 highlights the top 10 problems reported beginning in January 2000 and over the past two years including the last events analyzed above.

Events types are classified as death, injury or malfunction. Three death types were reported during this time period among 3 different manufacturers (Ventec Life Systems, Vyaire Medical and ResMed) and in each case there was no fault found in the ventilators and reporting entity states that the device was attached to the patient at the time of death.

Manufacturers involved in these 500 reports are arranged accordingly in figure 1. Device Brands involved in these 500 reports are arranged by event quantity are displayed in figure 2 . 


\begin{tabular}{|l|r|}
\hline PROBLEMS & 123 \\
\hline Output Problems & 102 \\
\hline Mechanical problem & 33 \\
\hline Circuit Failure & 33 \\
\hline Display & 30 \\
\hline No or Improper flow & 21 \\
\hline Power \& Electrical & 17 \\
\hline Connection problem & 14 \\
\hline Unexpected shutdown & 13 \\
\hline Degraded & 9 \\
\hline Alarm issue & 9 \\
\hline
\end{tabular}

Table 1: Top ten reported event device problems during the 15-day period

\begin{tabular}{|l|r|}
\hline PROBLEMS & 4617 \\
\hline Mechanical problem & 2975 \\
\hline Output Problems & 2403 \\
\hline Circuit Failure & 1611 \\
\hline Battery & 1554 \\
\hline Failure to recalibrate & 994 \\
\hline Protective Measures failure & 955 \\
\hline Failure of Device to self-test & 830 \\
\hline Failure to Charge & 775 \\
\hline No Display or Image & 742 \\
\hline Display or Visual Feedback & 7 \\
\hline
\end{tabular}

Table 2: Top 10 problems reported beginning in January 2000 and over the past two years including the last events analyzed above

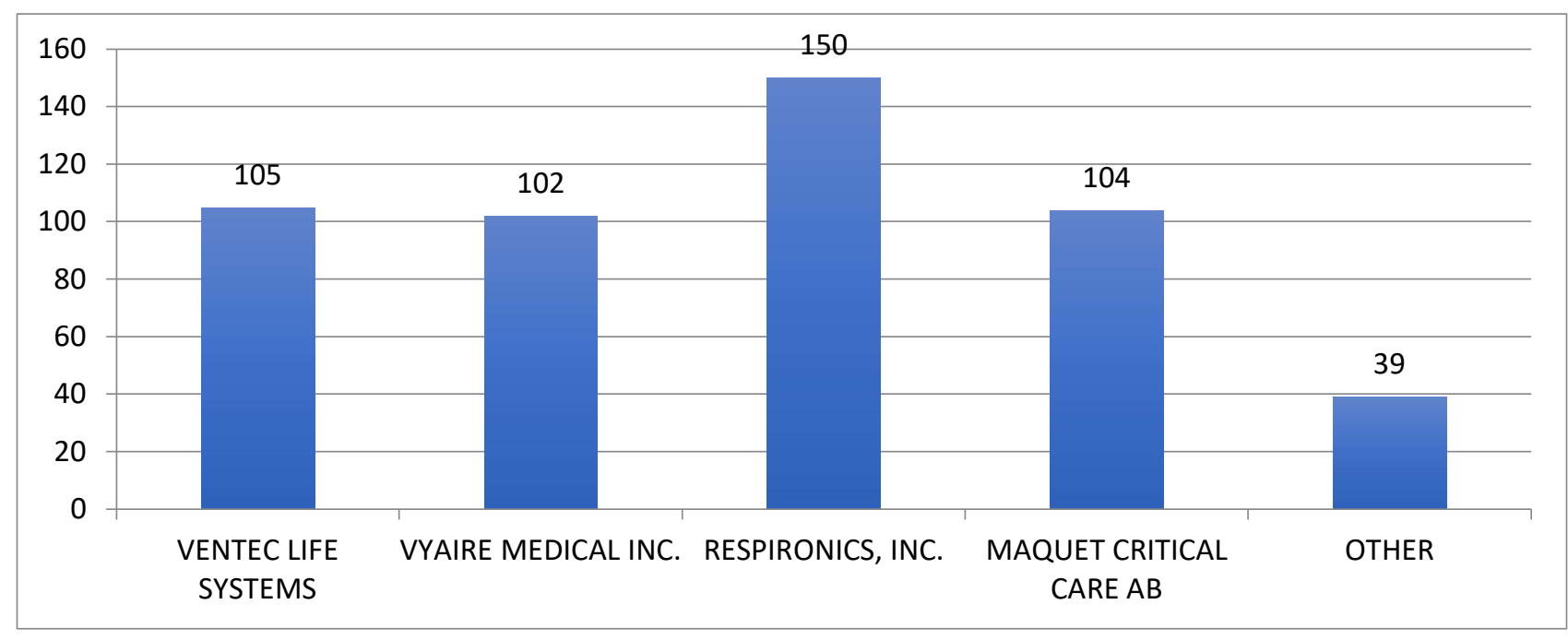

Figure 1: Manufacturers involved in the 500 reports are arranged accordingly 
Tunnell S A Review of Hospital based Ventilator Malfunctions Reported to the FDA in 2021

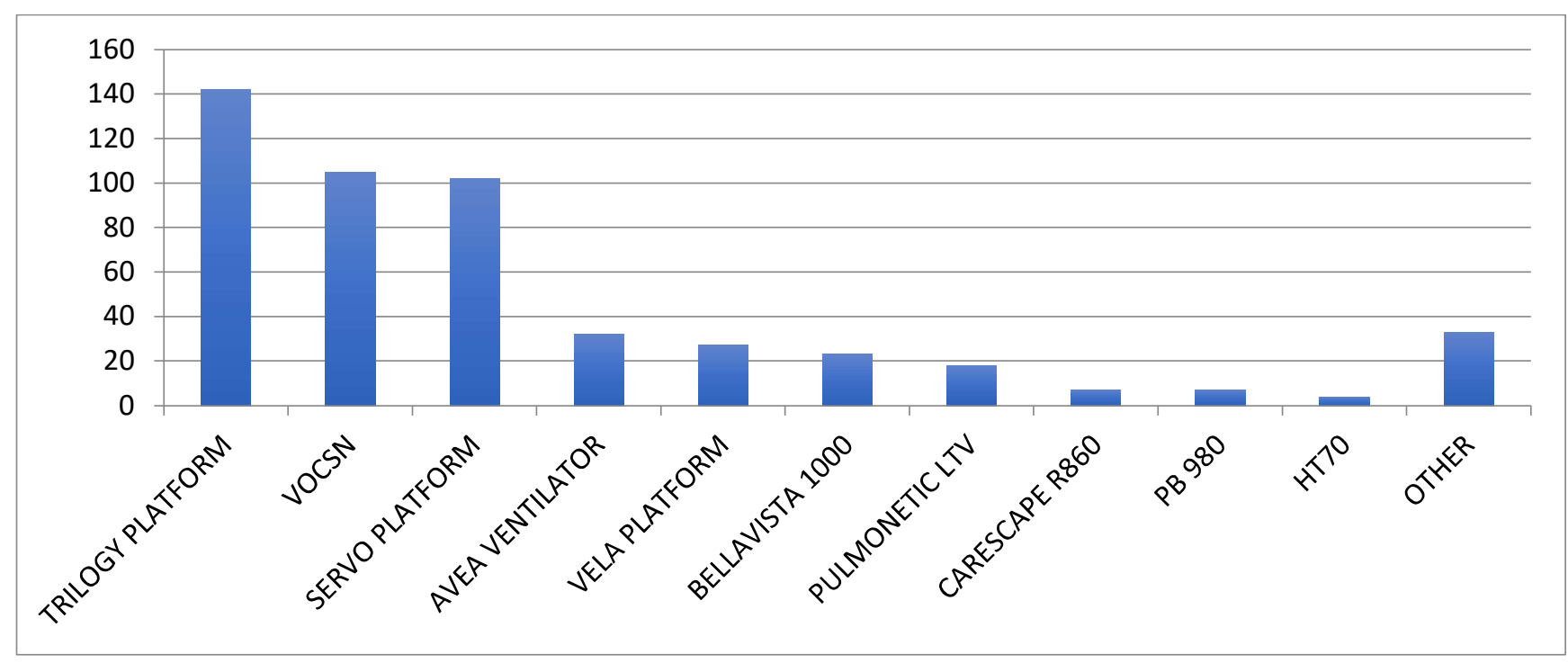

Figure 2: Device Brands involved in these 500 reports are arranged by event quantity

\section{Discussion}

It is important in the interpretation of incidence that one keeps in mind that devices with higher installed base would be more likely to have reported events.

Since the data collected is a mix of mandatory and voluntary submissions, it may include inaccuracies, and because the data is that of an initial report - without completed investigation, one must partially discount conclusions of lower statistical significance.

One must also broaden the scope of data analysis, including additional data if prevalence of a problem type is to be deduced. With that being said, it remains important for those who provide respiratory care and mechanical ventilation to be aware of this data and those in leadership positions should regularly examine this data.

In appraising the events found during this period of time some general themes can be deduced. The American Hospital Association claims 6009 acute care hospitals operating in the United States. ${ }^{3}$ They include Intensive care units that provide mechanical ventilation. Our current annualized rate of 2021 reported malfunction events are 12,167 . It can be easily calculated that there are on average 2 ventilator device malfunctions per year per acute care hospital. When accounting for Medical, Cardiac, Pediatric and Neonatal Intensive care beds ${ }^{3}$ in the US from the AHA data, we see there are 107,276 ICU beds. Therefore with 12,167 reported device malfunctions in an ecosystem where today, in COVID times, Intensive Care is sine qua non Mechanical Ventilation one can expect 1 in 10 ventilators per month to have a ventilator to human interpreted and reported malfunction.
On average each Acute Care Hospital will experience and have reported to the US FDA two continuous ventilator device malfunctions per anim. Despite the fact that this data used in this manner may lack some precision and specificity, I think we can all agree that this rate of interpreted malfunctions is too high.

It's understandable that a ventilator, a medical device, an electromechanical device would have consistently as the top two malfunctions, mechanical or output problems.

Electromechanical devices when they fail do so mechanically or electrically. Within the category of output malfunctions one sees that no patient injury or death are associated with these reported failures. In fact most were determined during startup testing and involved failures of blowers, pressure transducers, flow transducers or unresolved interface messages indicating failure.

Within the mechanical malfunctions reported, during the 15-day period, 10 patient injuries events were found. An examination of those injury events demonstrated that no patient injury actually occurred. Many of the failures occurred on patient. Many involved ventilators stopping or loosing function. A single manufacturer reported all 10 and an examination of other reports filed by different manufacturers with similar hazards events having occurred, indicated a different interpretation of injury reporting within companies.

In the entire 500 events clinical signs, symptoms or conditions were reported only reported $5 \%$ of the time and low oxygen saturation was a predominant reported sign. A single Cardiac Arrest and death was reported. 
An examination of Circuit problem events, which were the third most reported problem were isolated to a single manufacturer and they represented actual reported error codes during use indicating service needed and were not related to the ventilator circuit or breathing circuit. Those data are also available via MAUDE under the category code BZE.

A large amount of the reported events involves the interpretation of how the user believes the device is expected to behave and many do not take into account the expected intended functions of the device or use of the device. ${ }^{4}$

Because mechanical ventilators provide life sustaining care often people die on them. In this data transitions to home and changing of ventilator types were also noted as a hazard requiring practitioners understand the function of the device.

In addition to understanding as experts the devices employed, a practitioner needs to be aware of these publicly available databases so they can better understand tendencies and trends in their art.

\section{Conclusion}

Ventilator care is a primary tool for life support within the intensive care ward. This primary tool in the US involved both BioMed and Respiratory care services within the hospital to assure that these devices are fully operable and meet their intended use. The complexity of these devices and their associated controls and the interoperability between these devices and other devices occupying the breath delivery pathway require professional attention.

Between 2000 and 2001 we see a continued high use of mechanical ventilation and an alarming $9.6 \%$ increase in reported ventilator malfunctions resulting in projections of over 12,000 events being reported for 2021. It is important that the existing trends of reporting events to the FDA be continued.

There may be room for improvement in data requirements and guidance offered to classify patient injury. However, the availability of this information via the TPLC database publicly and in a timely manner serves as an important source for data on medical devices. Respiratory practitioners, physicians, nurses, risk managers and administrators must realize the importance of this data to inform them regarding their application of mechanical ventilation. ${ }^{5}$ With mortality rates for mechanical ventilation remaining high, those of us interested in the field of mechanical ventilation must also avail ourselves of these databases in order to move our practice forward.
Implications for change obviously include improved training to frontline practitioners regarding the use and functions of mechanical ventilators. Those who prepare ventilators for use should consider revising their existing performance protocols to elicit failure in the lab. Together with industry, well informed clinicians can make an impact on care.

\section{References}

1. Total Product Lifecycle database: https://www.accessdata.fda.gov/scripts/cdrh/cfdocs/cfTPL C/tplc.cfm

\section{MAUDE database:}

https://www.accessdata.fda.gov/scripts/cdrh/cfdocs/cfMA UDE/Search.cfm

3. American Hospital Association - Fast facts: https://www.aha.org/statistics/fast-facts-us-hospitals

4. Pham JC, Williams TL, Sparnon EM, et al. VentilatorRelated Adverse Events: A Taxonomy and Findings From 3 Incident Reporting Systems. Respir Care 2016;61(5):621-631.

5. Simonds AK. Risk management of the home ventilator dependent patient. Thorax 2006; 61:369-371.

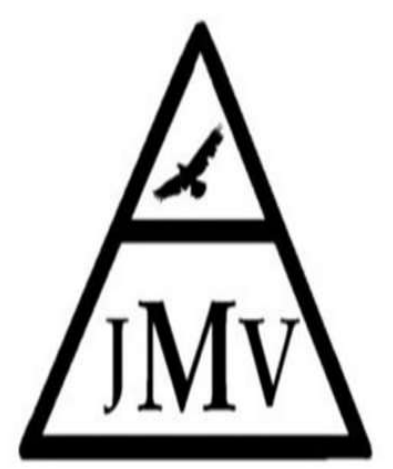

Journal of Mechanical Ventilation https://www.journalmechanicalventilation .com/submit-a-manuscript/
Free membership

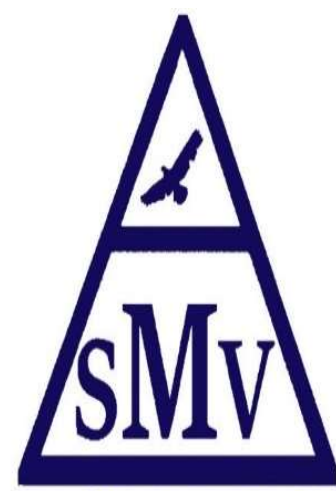

Society of Mechanical Ventilation

https://societymechanicalventilation.org /membership/ 